\title{
Composite Predictive Functional Control Strategies, Application to Positioning Axes
}

\author{
Jean-Yves Dieulot, Tarik Benhammi, Frédéric Colas, Pierre-Jean Barre
}

\begin{abstract}
Different predictive control strategies have been validated on a DC motor. ICascaded predictive control, which consists of a cascaded loop where the traditional servo algorithms are replaced by PFCs, could enhance the cycle time. Predictive Functional Control alone is simpler to tune and can exhibit comparable performances, except that the controller is more sensitive to nonlinear phenomena such as dry friction, which were not taken into account into the model and generate a static error.

Keywords: Model-based control, DC motor, Predictive control.
\end{abstract}

\section{Introduction}

The traditional cascaded-loop servo algorithm of a positioning device consists of a current, a speed and a position loop, for which the individual controllers within each loop use Proportional or PI algorithms [2,4]. This control structure is robust with respect to disturbances and easy to tune, loop after loop, when the inner loops dynamics are much faster than that of the outer loops. This is not always the case and the tracking lag may cumulates through the loops thus achieving poor performances.

Among various alternative control algorithms, Model-based Predictive Control which embeds both feedforward and a servo algorithms, is known for its tracking abilities - due to multiple-step-ahead prediction - and robustness with respect to model uncertainties [1]. In this paper, the focus will be on the implementation and fast tuning of the Predictive Functional Control Algorithm introduced by Richalet (e.g. $[7,8,9,10,12])$ which has been shown to be able to control a variety of complicated robot structures (see e.g. [11]). However, the main drawbacks of those Predictive Control methods are the need for a preliminary black-box model identification which hampers the implementation in real-time industrial world where tuning procedures should be simple and as short as possible.

Since a global controller may lose some of the advantages of cascaded loops, i.e. rejection of disturbances for each loop, dry friction compensation... . cascaded control structures have been introduced, where both position and speed loop can be replaced by a predictive controller. Since the PFC tracking performance is better, the lag in the inner loops should be reduced and better overall performances is assumed $[5,6]$.

In this paper, a comparison is drawn for different cascaded Predictive Functional Control strategies, which combines the PFC controller with a grey box model which parameters are related to physical characteristics.

\section{Predictive Functional Control}

\subsection{PFC philosophy}

In this section, the outline of model PFC algorithm, which is fully detailed in [7, 9] is recalled. PFC is based on a discrete linear model,

$$
\left\{\begin{array}{c}
x_{M}(n+1)=F_{M} x_{M}(n)+G_{M} u(n) \\
y_{M}(n)=C_{M}^{T} x_{M}(n)
\end{array}\right.
$$

$x_{M}$ is the state variable, $u n$ is the input, $y_{M}$ the system output, $F_{M}, G_{M}, C_{M}$ are constant matrices. The predictive control strategy is summed up in Fig. 1. A control sequence is designed to enforce the model 
output to stick to a reference trajectory $y_{R}$ at given coincidence points. Over this receding horizon, the reference trajectory which is the path to the future set point, is resetted at every instant and is given by:

$$
C(n+i)-y_{R}(n+i)=\alpha^{i} .\left(C(n)-y_{P}(n)\right), 0 \leqslant i \leqslant h
$$

where $C(n)$ is the set-point, $y_{p}(n)$ the real process output, $\alpha$ is a parameter $\alpha: 0 \leqslant \alpha \leqslant 1$, which represents the exponential convergence of the algorithm, and thus fixes the closed-loop behavior.

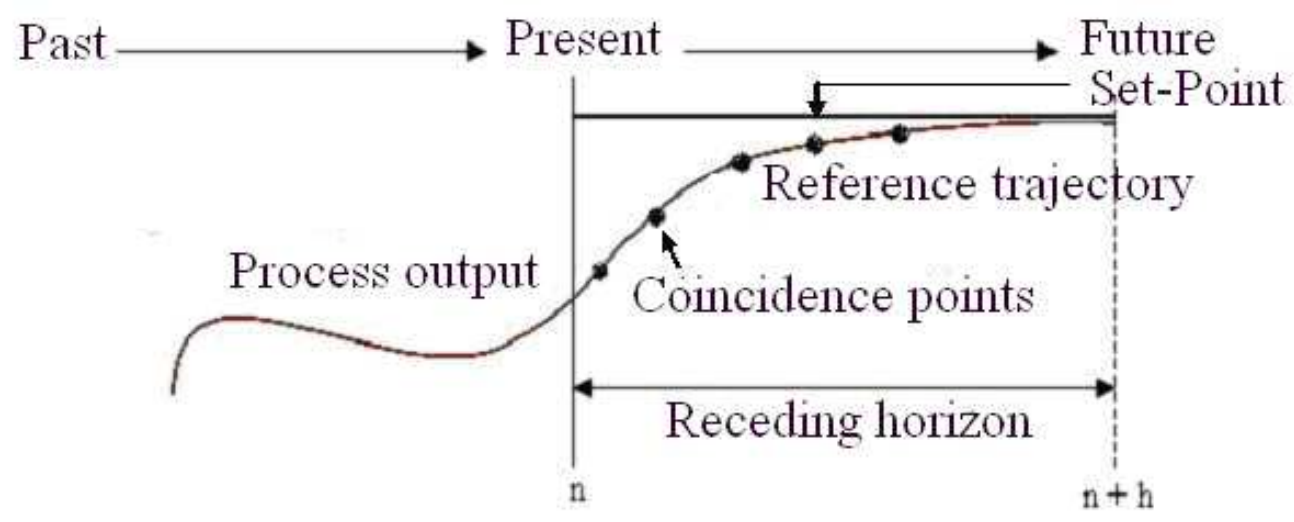

Figure 1: Principle of PFC control

\subsection{Computation of control}

The future controls sequence should optimize the performance index ((4)) which turns to find the minimum in the least-square sense of the tracking error at fixed set-points with respect to the future control sequence:

$$
D(n)=\sum_{j=1}^{n_{h}}\left\{\hat{y}_{P}\left(n+h_{j}\right)-y_{R}\left(n+h_{j}\right)\right\}^{2}
$$

where $h_{j}, 1 \leq i \leq h$ are the coincidence points.

In order to compute easily the control, it will be assumed that the latter is a composition of a priori orthonormal base functions (usually time-dependent polynomials $U_{B_{k}}(i)=i^{k-1}$ ),

$$
u(n+i)=\sum_{k=1}^{n_{B}}\left(\mu_{k}(n) U_{B_{k}}(i)\right), i \geq 0,
$$

where $U_{B_{k}}(i), 1 \leq k \leq n_{B}$ are the base functions, the optimization problem reducing to find the corresponding weights $\left.\mu_{(} k\right)$.

Of course, one could apply the whole control sequence, but, in fact, only the current control (5) is used, and the whole algorithm is computed again next step.

$$
u(n)=\sum_{k=1}^{n_{B}}\left(\mu_{k}(n) U_{B_{k}}(0)\right) .
$$

For computational purposes, the model output is divided into a free (unforced) response, where the control is set to zero, and a forced response to the control (4).

$$
y_{M}(n+i)=y_{F}(n+i)+y_{U F}(n+i), 1 \leqslant i \leqslant h
$$


where $y_{F}(n+i)=\sum_{k=1}^{n_{B}} \mu_{k}(n) \cdot y_{B_{k}}(i), 1 \leqslant i \leqslant h$ is the forced output, and $y_{B_{k}}$ is the response to the base function $u_{B_{k}}, y_{U F}(n+i)=C_{M}^{T} F_{M}^{i} x_{M}(n)$ is the unforced output. Let the predicted future error be approximated by a polynomial function:

$$
\hat{e}(n+i)=e(n)+\sum_{i=1}^{d_{e}} e_{m}(n) i^{m}
$$

where $h_{j}, 1 \leqslant j \leqslant n_{h}$ is a fixed approximation degree,

Finally, the controller is obtained by setting $\frac{\partial D(n)}{\partial \mu}=0$ (see [9], for details). The parameters only depend on the base functions, the convergence exponent and the coincidence points and can be computed off-line:

$$
u(n)=k_{0}\left\{c(n)-y_{p}(n)\right\}+\sum_{m=1}^{\max \left(d_{c}, d_{e}\right)} k_{m}\left\{c_{m}(n)-e_{m}(n)\right\}+v_{x}^{T} x_{M}(n)
$$

where $d_{c}$ is the degree of the polynomial approximation of the set-point and

$$
\begin{aligned}
& k_{0}=v^{T}\left[\begin{array}{c}
1-\alpha^{h_{1}} \\
1-\alpha^{h_{2}} \\
\vdots \\
1-\alpha^{h_{n_{h}}}
\end{array}\right], k_{m}=v^{T}\left[\begin{array}{c}
h_{1}^{m} \\
h_{2}^{m} \\
\vdots \\
h_{n_{h}}^{m}
\end{array}\right], v_{x}=\left[\begin{array}{c}
C_{M}^{T}\left(F_{M}^{h_{1}}-I\right) \\
C_{M}^{T}\left(F_{M}^{h_{2}}-I\right) \\
C_{M}^{T}\left(F_{M}^{h_{h_{h}}}-I\right)
\end{array}\right]^{-1} v \\
& v=\left(\sum_{j=1}^{n_{h}} y_{B}(h j) y_{B}(h j)^{T}\right)^{-1}\left[\begin{array}{lll}
y_{B}\left(h_{1}\right) & \ldots & \left.y_{B}\left(h_{h_{h}}\right)\right] U_{B}(0)
\end{array}\right.
\end{aligned}
$$

The algorithm consists thus of a feedforward term devoted for tracking error, a disturbance rejection term and of a state-space controller. In the application considered, control tuning will involve a "flat " prediction $(\hat{e}(n+i)=e(n))$ and a parameter $\alpha$, the parameters will be chosen with a rule of thumb [9] as

$$
\alpha=e^{\frac{-3 T e}{C L R T}}
$$

along with three coincidence points

$$
H=\left(\left[\begin{array}{lll}
C L R T / 3 & C L R T / 2 & C L R T
\end{array}\right] / T_{e}\right)
$$

where CLRT is the closed-loop rise time and $T_{e}$ is the sampling period.

\section{Industrial control and PFC strategies}

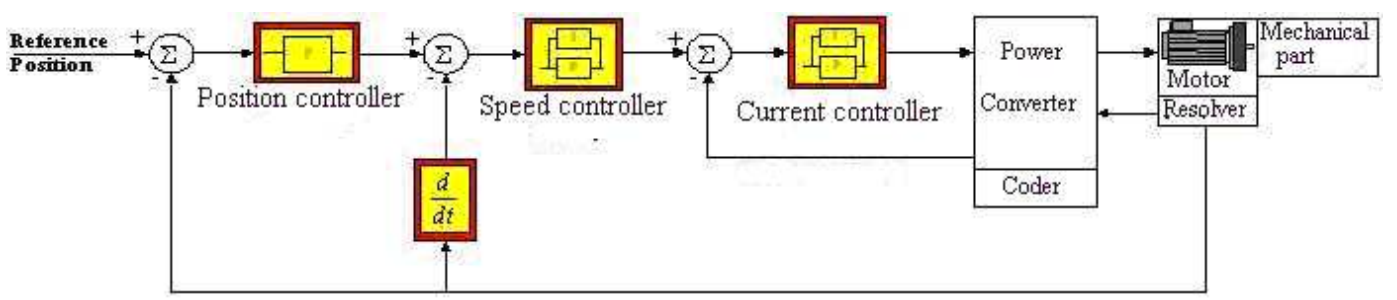

Figure 2: Full structure of the industrial cascaded-loop control

The industrial control consists of nested loops and assumes that the dynamics of the inner loops are the fastest ; the current loop has generally a high dynamics and is thus neglected, the speed loop involves 
a PI structure and the position loop only a proportional controller, and sometimes a low-pass filter which has been shown to improve the dynamics of axes, particularly in presence of flexibilities. Traditionally, the set-point is a bang-bang in acceleration which takes in account limitations in speed and acceleration and is a time-optimal trajectory. As was explained in introduction, this controller has an easy step-bystep tuning procedure, from the fastest to the slowest loop, and saturations, disturbances can be easily handled at every loop level. However, it is known that performances are not as good as those of a global controller, because the tracking lag is cumulating over the successive loops. The speed controller is simply $C(s)=k_{v}\left(1+\frac{1}{\tau_{v} s}\right)$ where $k_{v}, \tau_{v}$ are the speed loop proportional and integral gains.

As an alternative to this cascaded controller or to a global controller (such as a PFC), a composite cascaded structure where the speed loop PI controller is replaced by a speed loop PFC controller, hence realizing the decoupling of the different dynamics (Fig. 3).

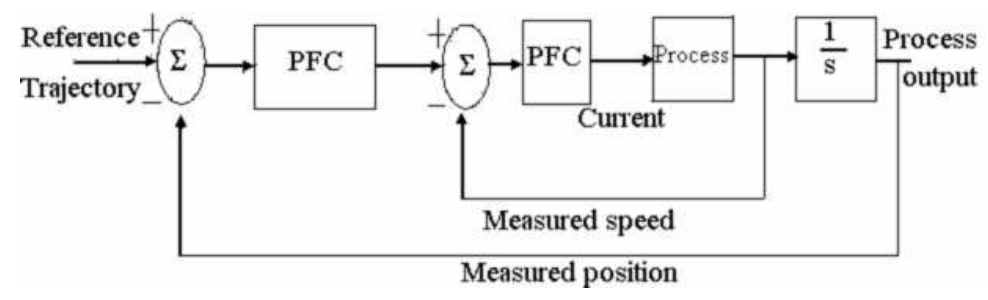

Figure 3: Composite cascaded controller

The last scheme consists of the introduction of a cascaded PFC control which is the same as the classical industrial controller except that PFC servo algorithms are embedded into both loops (Fig. 4).

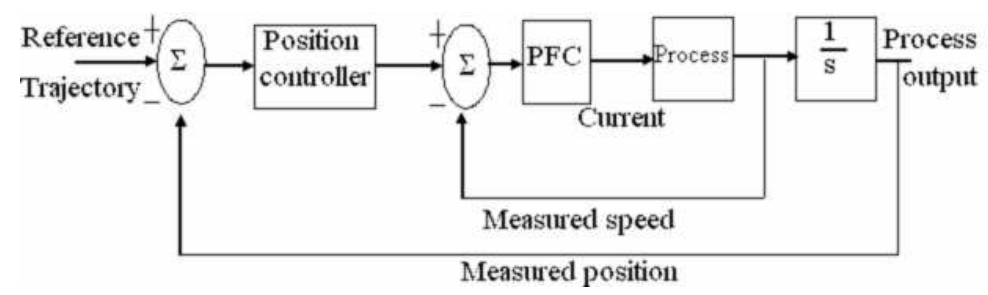

Figure 4: Cascaded PFC controller

\section{Modelling and experimentation}

The experimental set-up is a brushless DC servo-motor (PARVEX F9M-4-57), equipped with a tacho-generator (F9TS-7-270) and a position coder (resolver), which drives a rotating shaft mounted on bearings, optionnally coupled to an additional flywheel.

When neglecting the electrical resistances, the global model of the brushless motor is:

$$
J_{e q} \frac{d \omega}{d t}=C_{m}-f_{v} \omega, C_{m}=K_{t} i
$$

where $\omega$ is the rotating speed, $C_{m}$ and $i$ are the control torque and current, $K_{t}$ is the torque constant, $J_{e} q$ is the equivalent motor inertia, $f_{v}$ is the viscous damping coefficient.

Most PFC algorithms require a proper identification and modeling stage; the motor inertia was estimated from the known motor and load moment of inertia which were added to the inertia resulting from the transmission coupling. Only the viscous and dry friction coefficients were estimated experimentally from a plot of the motor current versus the shaft rotation speed. Characteristics are summed up in Table 1 . 


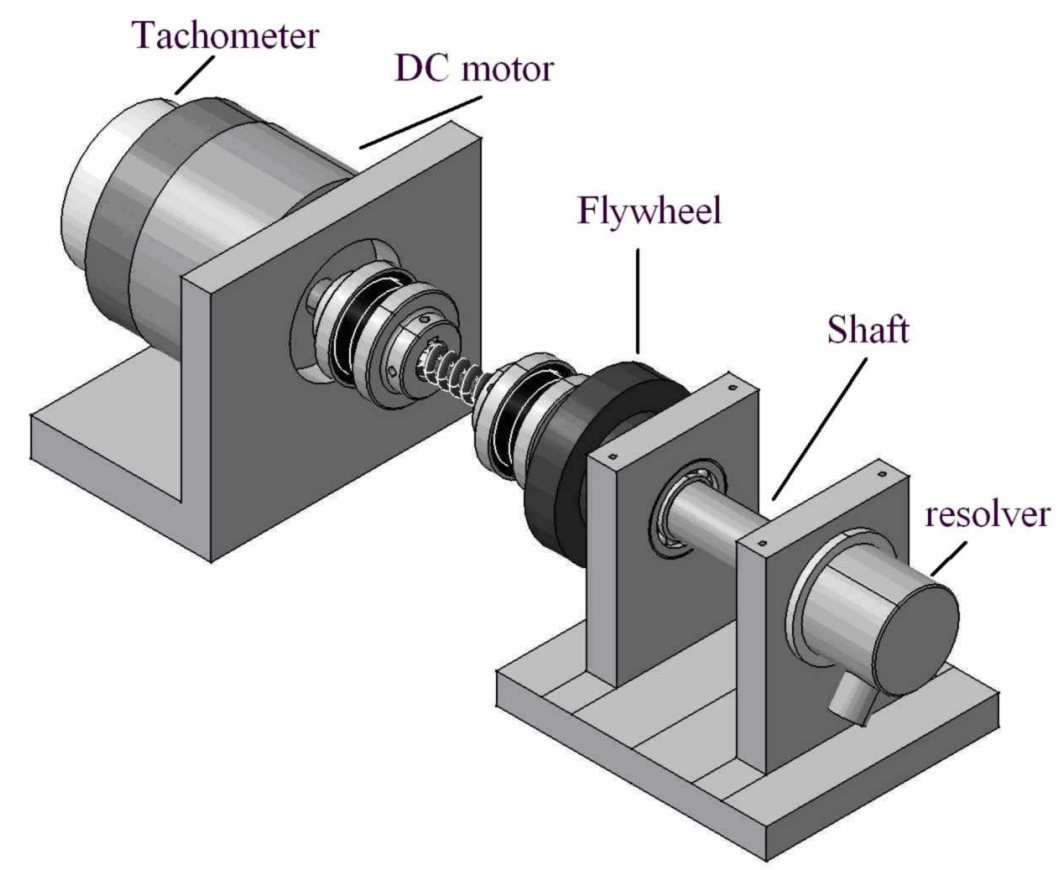

Figure 5: Experimental DC motor

\begin{tabular}{|l|l|l|}
\hline Controller & $K_{p}\left(\mathrm{rad} . \mathrm{A}^{-1}\right)$ & $C L R T(\mathrm{~s})$ \\
\hline PFC & N/A & 0.02 \\
\hline Cascaded PFC & N/A & $\begin{array}{l}\text { Speed loop 0.01 Position } \\
\text { Loop 0.06 }\end{array}$ \\
\hline Composite PFC & 10 & 0.05 \\
\hline Industrial control & 10 & N/A \\
\hline
\end{tabular}

Table 1: Motor characteristics 


\begin{tabular}{|l|l|l|l|l|l|}
\hline $\begin{array}{l}\text { Nominal } \\
\text { Torque N.m }\end{array}$ & $\begin{array}{l}\text { Nominal } \\
\text { Speed } \\
\text { Tr.min }^{-1}\end{array}$ & $\begin{array}{l}\text { Electric } \\
\text { Power W }\end{array}$ & $\begin{array}{l}\text { Equivalent } \\
\text { inertia } \\
\text { Kg. } \mathrm{m}^{2}\end{array}$ & $\begin{array}{l}\text { Flywheel in- } \\
\text { ertia Kg.m }{ }^{2}\end{array}$ & $\begin{array}{l}\text { Viscous } \\
\text { damping cf. } \\
\text { Nmsrad }^{-1}\end{array}$ \\
\hline 0.346 & 3000 & 108 & $1.5 .10-4$ & $8.5 .10-4$ & 7.8 \\
\hline
\end{tabular}

Table 2: Control parameters

The measure of speed was low-pass filtered with a filter cut-off frequency of $2 \cdot 10^{-3} \mathrm{~s}$. Measurements recording and real-time control were achieved using a DSpace 1103 board with a sampling period of $T_{e}=5.10^{-4} \mathrm{~s}$. The experimental reference position was set to $12 \mathrm{rad}$, the maximum speed $V_{m} a x$ to 150 $\mathrm{rads}^{-}$, the maximum acceleration to $A_{\max }=346 \mathrm{rads}^{-2}$. The traditional trajectory for positioning axes consists of a time-optimal controller [3] with constrained speed and acceleration. The control gains for each experiment are given in Table 2. The integral gain of the PI speed is calculated to compensate the first-order pole, with $\tau_{v}=\frac{f_{v}}{J_{e q}}$, the speed gain $K_{v}$ is tuned so that the control torque does not exceed the peak torque, which gives $K_{v}=1$ rad.s. $\mathrm{A}^{-1}$. PFC algorithms were tuned after performing simulations in a way that the maximum current does not overcome that of the industrial loop.

\section{Experimental Results and discussion}

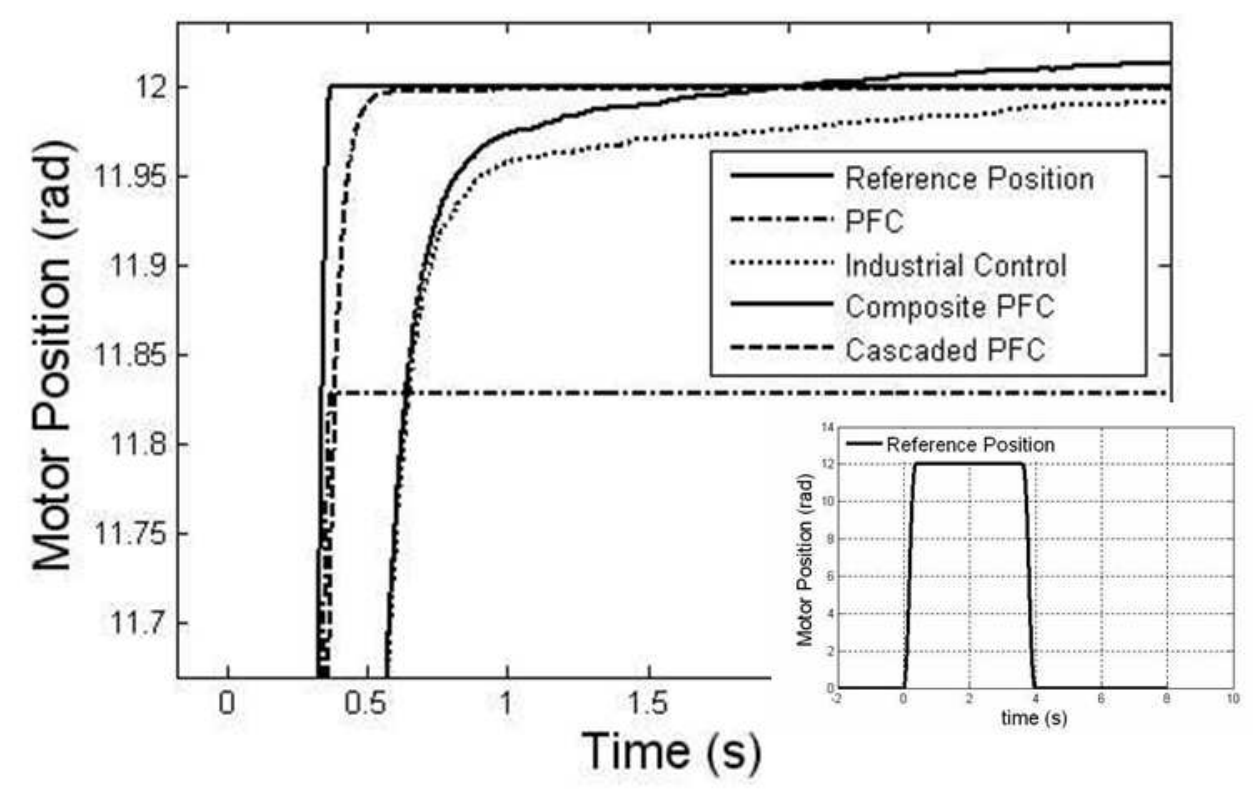

Figure 6: Comparison of control performances

Fig. 6 shows the basic results that will serve as a comparison of the different control structures: one can see that a global controller such as a PFC or a controller with a fast closed-loop position controller exhibits a small rise time due mainly to the cancellation of the phase lag in the speed loop and in the position loop. The performances of PFC controllers outperform that of the traditional industrial control, for a comparable current maximum amplitude. However, these controllers have two main drawbacks: the PFC has an important static error because the dry friction is not taken into account into the model. This phenomenon does not occur with the cascaded loop because these errors appear firstly in the speed loop, and can then be compensated with the position loop as in traditional control. Cascaded PFC seems 
to be the most appealing, but one has to remind that the tuning is far more delicate because it involves two PFC loops. One should take an appropriate CLRT ratio between the loops (at least 5) in a way that the position loop be truly the fastest.

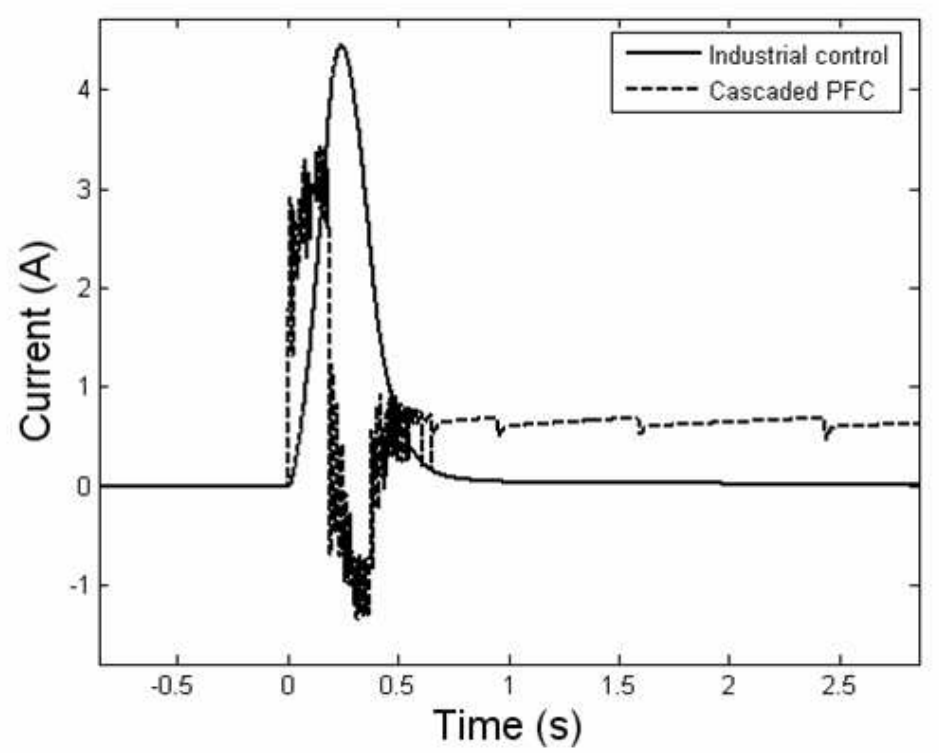

Figure 7: Drive current - control -

Fig. 7 shows that, for cascaded PFC -as for other PFC algorithms - , less energy may be needed, but the current signal is less smooth than with an industrial control scheme; clearly, this could excite vibration modes when a very flexible load is carried.

Most controls are robust when the additional inertia has been removed, only the rise-time is a little higher. The composite controller does not improve the industrial loop by much, except when an external disturbance is applied (e.g. braking with the hand) - results are not shown here -, with a better rejection. In this case, classical PFC is the less robust with respect to other controls.

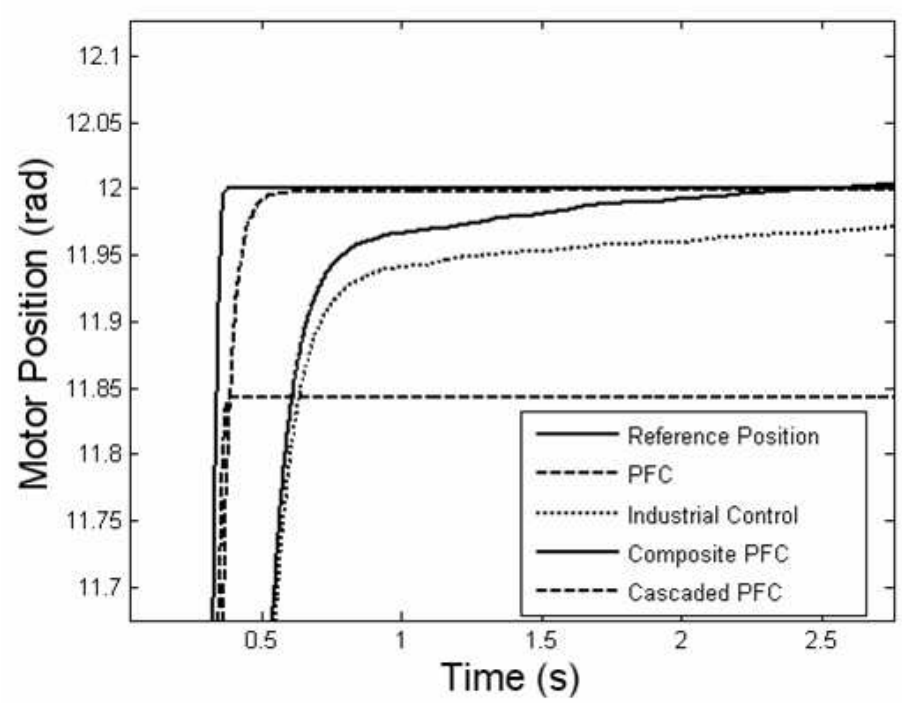

Figure 8: Control Performances with no load 


\section{Conclusion}

Composite Predictive Functional Control algorithms - a cascaded structure where only the speed loop servo algorithms or both speed and position controllers were replaced by PFCs - were validated on a DC servo-motor. The tuning procedure was quite simple and involved the general rigid model which can be found from the physical characteristics of the motor and a single experiment (for the determination of the viscous friction parameter). Cascaded predictive control could improve the tracking and was shown to be more robust to load mass variations. Classical PFC is easier to tune (only one PFC loop), the closed-loop performances being nearly the same; however, the controller is more sensitive to unmodelled nonlinear phenomena such as dry friction, which were not taken into account into the model. The resulting static error can be unacceptable and alternative solutions have, in this case, to be proposed. Future work will focus on application to flexible manipulators.

\section{Bibliography}

[1] Clarke, D. W., Mohtadi, C.,Tuffs, P. S. Generalized predictive control. Part I: the basic algorithm. Part II: Extensions and interpretations, Automatica, Vol. 23, 1987, pp 137 - 160.

[2] Ellis, G., Control system design guide (2nd edition), Academic Press, Boston, 2000.

[3] Erkorkmaz, K., Altintas, Y., High-speed CNC system design. Part III: high speed tracking and contouring control of feed drives, Int. J. Machine Tools \& Manuf., Vol. 41, 2001, pp. 1637 - 1658.

[4] GroSS, H., Harmann, J., Wiegârtner, G., Electrical feed drives in automation, MCD corporate Publishing, SIEMENS, ISBN 3-89578-148-7, 2001.

[5] Guemghar, K., Srinivasan, B., Mullhaupt, Ph., Bonvin, D., Analysis of cascade structure with predictive control and feedback linearisation, IEE Proceedings-Control Theory and Applications, Vol. 152, 2005, pp $317-324$.

[6] Hedjar, R., Toumi, R., Boucher, P., Dumur, D., Two cascaded nonlinear predictive controls of induction motor, Proceedings of 2003 IEEE Conference on Control Applications., 2003

[7] Richalet, J., Industrial applications of model-based predictive control, Automatica, Vol. 29, 1993, pp $1251-1274$.

[8] Richalet, J., Estival, J.L., Fiani, P., Industrial applications of predictive functional control to metallurgical industries, Proc. 4th IEEE Conf. Control Applications, 1995.

[9] Richalet, J., Abu, E., Arber C., Kuntze, H.B., Jacubasch, A., Schill, W., Predictive functional control. Application to fast and accurate robot, 10th IFAC World Congress, Munich, Germany, 1997

[10] Rossiter, J. A., Predictive functional control: more than one way to prestabilise, 15th IFAC World Congress. Barcelona, Spain, 2002.

[11] Vivas, A., Poignet, P., Predictive functional control of a parallel robot, Control Engineering Practice, Vol. 13, 2005, 863 - 874.

[12] Yim W., Singh S. N., Nonlinear inverse and predictive end point trajectory control of flexible macromicro manipulators, J. Dyn. Dyst. Meas. Control, Vol. 119, 1997, pp 412 - 420. 
Jean-Yves Dieulot ENSAM de Lille

Research Technological Team CEMODYNE

8 bd Louis XIV, 59000 LILLE Cedex E-mail: jean-yves.dieulot@polytech-lille.fr

Tarik Benhammi ENSAM de Lille

Research Technological Team CEMODYNE 8 bd Louis XIV, 59000 LILLE Cedex E-mail: jean-yves.dieulot@polytech-lille.fr

Frédéric Colas ENSAM de Lille

Research Technological Team CEMODYNE 8 bd Louis XIV, 59000 LILLE Cedex

E-mail: frederic.colas@centraliens-lille.org

Pierre-Jean Barre ENSAM de Lille

Research Technological Team CEMODYNE 8 bd Louis XIV, 59000 LILLE Cedex E-mail: barre@ lille.ensam.fr Received: November 6, 2007 


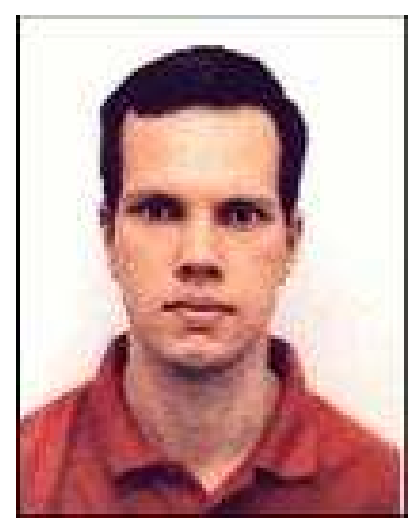

Jean-Yves Dieulot graduated from French Ecole d'Ingénieurs Institut Industriel du Nord in 1990, and obtained a PhD from the University of Lille in 1993. He is currently Associate Professor at Ecole Polytechnique Universitaire de Lille, France, and with LAGIS (Laboratory of Automatic Control) His main interests are in fuzzy, nonlinear and robot control.

Tarik Benhammi graduated from Ecole Nationale Supérieure des Arts et Métiers, in Meknès, Morocco, in 2007.

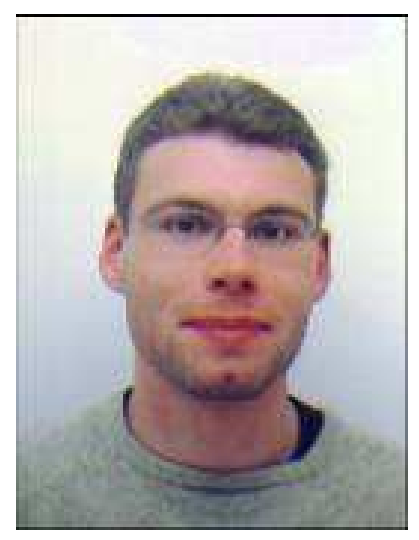

Frédéric Colas graduated from Ecole Centrale de Lille, France, in 2003. He has obtained a PhD from Ecole Centrale de Lille, France, in Robotics and Automatic Control, which was funded by cartesian robot manufacturers.

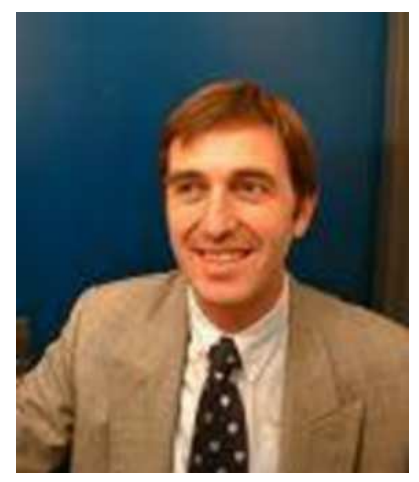

Pierre-Jean Barre is a Full Professor and Head of Ecole Nationale Supérieure des Arts et Métiers, in Lille, since 2004. He has also developed a Technological Research Platform devoted to highspeed flexible robot control and planning, funded by many French and European manufacturers. 